\title{
Nerve Tissue and Nerve Sheaths
}

National Cancer Institute

\section{Source}

National Cancer Institute. Nerve Tissue and Nerve Sheaths. NCI Thesaurus. Code

C41443.

Tissues that contain neurons and supporting cells that form the covers encircling nerves, nerve fibers, and axons. 